\title{
Hypertriglyceridemic Waist - a Simple Clinical Tool to Detect Cardiometabolic Risk: Comparison With Harmonized Definition of Metabolic Syndrome
}

\author{
H. VAVERKOVÁ ${ }^{1}$, D. KARÁSEK ${ }^{1}$, D. NOVOTNÝ ${ }^{2}$, M. HALENKA ${ }^{1}$, J. ORSÁG ${ }^{1}$, \\ L. SLAVÍK ${ }^{3}$
}

${ }^{1}$ Third Department of Internal Medicine - Nephrology, Rheumatology and Endocrinology, Faculty of Medicine and Dentistry, Palacky University, Olomouc, Czech Republic, ${ }^{2}$ Department of Clinical Biochemistry, University Hospital, Olomouc, Czech Republic, ${ }^{3}$ Department of Hemato-Oncology, University Hospital Olomouc, Czech Republic

Received September 21, 2015

Accepted October 5, 2015

\begin{abstract}
Summary
With the increasing prevalence of obesity and especially abdominal obesity, a simple clinical tool is needed that identifies the cardiometabolic risk for cardiovascular disease and type 2 diabetes. The aim of our study was to evaluate a broad spectrum of metabolic variables and IMT in subjects with and without hypertriglyceridemic waist (HTGW) and compare it with the harmonized definition of metabolic syndrome (MS) with both a higher (MS-I) and lower waist circumference (MS-II) for Europids. We enrolled 607 asymptomatic dyslipidemic subjects (295 men and 312 women) into our cross-sectional study. The subjects with HTGW had an atherogenic lipid profile (significantly higher triglycerides, AIP, non-HDL-C, lower HDL-C and ApoA-1, and the women also higher TC and $A$ poB), increased markers of insulin resistance (insulin, HOMA, C-peptide, proinsulin), inflammation (hsCRP), thrombosis (fibrinogen, PAI-1), SBP and DBP, and lower adiponectin ( $p<0.05-0.001$ for all). These risk factors were entirely similar in HTGW, MS-I and MS-II. Age-adjusted IMT was significantly higher only in the women with HTGW but this significance disappeared after further adjustment for TC, SBP, and smoking. Our results support the routine use of HTGW as a simple and inexpensive screening tool to detect subjects at increased cardiometabolic risk in clinical practice.
\end{abstract}

\section{Key words}

Hypertriglyceridemic waist • Metabolic syndrome • Lipid metabolism • Thrombosis • IMT

\section{Corresponding author}

H. Vaverková, Third Department of Internal Medicine Nephrology, Rheumatology and Endocrinology, University Hospital Olomouc, I. P. Pavlova 6, 77900 Olomouc, Czech Republic. Fax:+420588442526. E-mail: helena.vaverkova@fnol.cz

\section{Introduction}

With the increasing prevalence of obesity in populations throughout the world and the changing of risk factors towards more abdominal obesity and subsequent insulin resistance (Bruthans et al. 2014), a simple clinical tool is needed that identifies cardiometabolic risk for diseases such as cardiovascular disease and type 2 diabetes.

The concept of hypertriglyceridemic waist (HTGW) as a simple phenotype to detect subjects with cardiometabolic risk was suggested 15 years ago by Lemieux et al. (2000). They were the first group to recognize that hypertriglyceridemia plus a large waist circumference (WC) (hypertriglyceridemic waist, HTGW) is associated with a metabolic triad of unconventional risk variables (hyperinsulinemia, hyperapolipoprotein B, and small dense LDL). This atherogenic metabolic triad was associated with a more than 20-fold increase in the risk of ischemic heart disease in middle-aged men enrolled in the Quebec Cardiovascular Survey, beyond the presence of traditional risk factors (Lamarche et al. 1998). Lemieux et al. (2007)

PHYSIOLOGICAL RESEARCH • ISSN 0862-8408 (print) • ISSN 1802-9973 (online)

(c) 2015 Institute of Physiology v.v.i., Academy of Sciences of the Czech Republic, Prague, Czech Republic

Fax +420 241062 164, e-mail: physres@biomed.cas.cz, www.biomed.cas.cz/physiolres 
also suggested that the HTGW phenotype is a central component of metabolic syndrome.

The definition of metabolic syndrome, depending on the presence of at least three out of five markers, was proposed by many expert groups until a harmonized definition of MS was recently accepted (Alberti et al. 2009). This definition is also supported by the ESC/EAS guidelines for the management of dyslipidemias (Reiner et al. 2011).

The aim of our study was to compare a broad spectrum of metabolic variables (atherogenic lipid profile, markers of insulin resistance, inflammation, thrombosis and fibrinolysis, and adiponectin) in asymptomatic dyslipidemic subjects with and without HTGW and compare it with the last harmonized definition of MS. In a subset of patients we also evaluated intima media thickness (IMT) as a marker of subclinical atherosclerosis.

\section{Materials and Methods}

\section{Study design and subjects}

The study was carried out as a cross-sectional study on asymptomatic dyslipidemic subjects who had been examined at the Lipid Center of the $3^{\text {rd }}$ Department of Internal Medicine, University Hospital Olomouc, Czech Republic. All the dyslipidemic subjects filled out a questionnaire on their medical history, especially their cardiovascular status, medication and smoking habits. All the subjects were tested for an underlying cause of secondary hyperlipidemia: diabetes mellitus, hypothyroidism, hepatic or renal impairment, and nephrotic syndrome. Subjects with these diagnoses were not enrolled in the study. Other exclusion criteria were a history of clinically manifest atherosclerosis (coronary artery disease, cerebrovascular ischemic disease, and peripheral arterial disease), hypolipidemic treatment in the previous six weeks, and the clinical presence of acute infectious disease or trauma. Dyslipidemia was defined as total cholesterol $\geq 5 \mathrm{mmol} / \mathrm{l}$ and/or triglycerides $(\mathrm{TG}) \geq$ $1.5 \mathrm{mmol} / \mathrm{l}$. 607 subjects (295 men and 312 women) fulfilled the above-mentioned criteria and were included in the study. In a subset of 369 patients $(60.8 \%$ 173 males and 196 females) IMT measurement was performed.

The study was reviewed and approved by the Institutional Ethics Committee of the Medical Faculty and University Hospital and informed consent was obtained from all participants.

\section{Anthropometric and laboratory measurements}

The WC was measured in the standing position, at the middle point between the anterior iliac crest and the lower border of the ribs. The body mass index (BMI), waist circumference (WC), systolic (SBP), and diastolic (DBP) were determined. The auscultatory method of BP measurement with a properly calibrated and validated mercury sphygmomanometer was used. At least three sitting BP measurements were taken at 30-s intervals and the mean of the last two was calculated. Patients treated with antihypertensive drugs or with $\mathrm{SBP} \geq 140$ or DBP $\geq$ $90 \mathrm{~mm} \mathrm{Hg}$ were assumed to be hypertensive.

The hypertriglyceridemic waist phenotype was defined as waist $\geq 90 \mathrm{~cm}$ and $\mathrm{TG} \geq 2.0 \mathrm{mmol} / 1$ in men, and waist $\geq 85 \mathrm{~cm}$ and $\mathrm{TG} \geq 1.5 \mathrm{mmol} / \mathrm{l}$ in women (Arsenault et al. 2010).

For a diagnosis of MS we used a harmonized definition (Alberti et al. 2009) with both the higher (MS-I) and lower (MS-II) WC thresholds for Europids (MS-I: $W C \geq 102 \mathrm{~cm}$ in men and $\geq 88 \mathrm{~cm}$ in women; MS-II: $W C \geq 94 \mathrm{~cm}$ in men and $\geq 80 \mathrm{~cm}$ in women). The presence of any three of five risk factors constitutes a diagnosis of MS: elevated WC (see above); triglycerides $\geq 1.7 \mathrm{mmol} / \mathrm{l}$ (drug treatment for elevated $\mathrm{TG}$ is an alternative indicator - not present in our cohort); reduced HDL-C $<1.0 \mathrm{mmol} / 1$ in men and $<1.3 \mathrm{mmol} / 1$ in women (drug treatment for reduced HDL-C is an alternative indicator - not present in our cohort); elevated blood pressure (systolic $\geq 130$ and/or diastolic $\geq 85 \mathrm{~mm} \mathrm{Hg}$ or antihypertensive drug treatment in a patient with a history of hypertension), and elevated fasting glucose $\geq 5.6 \mathrm{mmol} / 1$ (drug treatment of elevated glucose is an alternative indicator - not present in our cohort).

\section{Biochemical analyses}

Venous blood samples were drawn in the morning after a 12-h fast. Total cholesterol (TC), triglycerides (TG), and HDL-cholesterol (HDL-C) were determined enzymatically on a Modular SWA analyzer (Roche, Basel, Switzerland) using commercially available kits (Cholesterol SYS 917, Triglycerides GPO-PAP and HDL cholesterol plus, third-generation kits, Roche, Basel, Switzerland). The determination of HDL-C was performed by a direct method without precipitation of lipoproteins containing apoB. LDL-C levels were calculated according to the Friedewald formula (for TG $<4.5 \mathrm{mmol} / \mathrm{l})$ in subjects with $\mathrm{TG} \leq 4.5 \mathrm{mmol} / \mathrm{l}$. We also calculated the non-HDL cholesterol (non-HDL-C $=\mathrm{TC}-$ 
HDL-C) and atherogenic index of plasma AIP = $\log$ (TG/HDL-C). The concentration of apolipoprotein B (ApoB) and apolipoprotein A1 (apoA1) was determined immunoturbidimetrically on a Modular SWA analyzer (TinaQuant Apo A1, TinaQuant Apo B kits, all Roche, Basel, Switzerland). Lipoprotein (a) [Lp(a)] was determined immunoturbidimetrically using a Lipoprotein (a) Tina-Quant TQ kit (Roche, Basel, Switzerland). High-sensitivity C-reactive protein (hs-CRP) was assessed by means of an ultra-sensitive latex immunoturbidimetric method on a Modular SWA analyzer (CRP latex TinaQuant kit, all Roche, Basel, Switzerland). Glycemia was determined by means of the enzymatic-colorimetric method (Glucose GOD-PAP kit) on a Modular SWA analyzer. Insulin was determined using commercially available kits - Insuline (Immunotech, Marseille, France) using specific antibodies by the IRMA (immunoradiometric assay) method. The result obtained was then used for the calculation of the parameter of insulin resistance HOMA [homeostasis model assessment, described by Matthews et al. (1985): fasting glycemia $(\mathrm{mmol} / \mathrm{l}) *$ fasting insulin $(\mathrm{mU} / 1) / 22.5]$. C-peptide and intact proinsulin were determined using commercially available kits - C-peptide (Immunotech, Marseille, France) and Proinsulin (DRG Instruments $\mathrm{GmbH}$, Marburg, Germany) using specific antibodies by the IRMA method for C-peptide and the radioimmune assay (RIA) method for proinsulin. Concentrations of insulin, proinsulin, C-peptide, and hs-CRP were measured in the serum stored at $-80{ }^{\circ} \mathrm{C}$. For the assessment of prothrombotic markers venous blood was collected in $3.8 \%$ sodium citrate tubes (dilution 1:10). The following markers were examined: fibrinogen with the TC Thrombin Reagent (Technoclone, Vienna, Austria) using the method according to Clauss on an ACL Top coagulation analyzer (Werfen, Milano, Italy) from fresh plasma, the von Willebrand factor immunoturbi-dimetric assay (Instrumentation Laboratory Spa, Milan, Italy), plasminogen activator inhibitor-1 and tissue plasminogen activator - ELISA (Technoclone, Vienna, Austria).

The total adiponectin was measured in serum (one separate aliquot stored at $-80{ }^{\circ} \mathrm{C}$ until the day of analysis) with Human Adiponectin ELISA imunochemical kit (Biovendor Laboratory Medicine Inc., Brno, Czech Republic), according to the manufacturer's instructions.

\section{IMT measurement}

IMT measurement was performed in a subset of 369 patients (60.8\% - 173 males and 196 females). Ultrasound scanning of IMT was performed with a 10-MHz linear array transducer (Philips Sonos 5500, 2004). All the measurements were performed with the subjects in a supine position. The head was tilted to one side at an angle of $45^{\circ}$. The longitudinal B-mode image of the common carotid artery (CCA) was displayed just before the widening of the bulb. When an optimal longitudinal image of the far wall of the CCA in the region of $1 \mathrm{~cm}$ proximally from the bulb was obtained, it was frozen on the $\mathrm{R}$ wave according to a simultaneous ECG and videotaped. Three video recordings were made on both CCA. The IMT measurements were processed off-line using the Image-Pro Plus software (Version 4.0, Media-Cybernetics, Silver Spring, USA). The region under evaluation was the CCA wall $1-2 \mathrm{~cm}$ distant proximally from the above-mentioned border. The average of all the mean IMT of three frozen images of both sides was chosen as the outcome variable. The measurement of IMT was performed without knowledge of the laboratory results.

\section{Statistical analyses}

All the values are expressed as means $\pm \mathrm{SD}$ or as median for variables with non-normal distribution. The Kolmogorov-Smirnov test was used to test for normal distribution. Variables with a non-normal distribution (hsCRP, TG, insulin, HOMA, C-peptide, proinsulin, fibrinogen, t-PA, PAI-1, adiponectin) were logtransformed to normalize their distribution before statistical analysis. The differences in the means between the groups were analyzed using ANKOVA after adjustment for age in both sexes. IMT was further adjusted for other variables incorporated in the SCORE system (total cholesterol, systolic blood pressure, and smoking). Differences in categorical variables were analyzed by means of $\chi^{2}$ test. Statistical analysis was performed using SPSS for Windows version 12.0 (Chicago, Illinois, USA). Probability values of $\mathrm{p}<0.05$ were considered statistically significant.

\section{Results}

The characteristics of the dyslipidemic subjects without (0) and with (1) HTGW, MS-I, and MS-II are summarized in Table 1 for the male subjects and in Table 2 for the female subjects. 
Table 1. Physical characteristics and cardiometabolic risk profile of MALE dyslipidemic subjects with (1) and without (0) HTG W, MS-I and MS-II.

\begin{tabular}{|c|c|c|c|c|c|c|}
\hline Parameter & $\begin{array}{c}\text { HTGW (0) } \\
\text { n=160 }\end{array}$ & $\begin{array}{c}\text { HTGW (1) } \\
\text { n=135 }\end{array}$ & $\begin{array}{c}\text { MS-I (0 } \\
\text { n=184 }\end{array}$ & $\begin{array}{c}\text { MS-I (1) } \\
n=111\end{array}$ & $\begin{array}{c}\text { MS-II (0) } \\
\text { n=158 }\end{array}$ & $\begin{array}{c}\text { MS-II (1) } \\
\mathbf{n}=\mathbf{1 3 7}\end{array}$ \\
\hline Age (years) & $41.9 \pm 15.4$ & $46.6 \pm 11.1^{* * *}$ & $41.6 \pm 14.5$ & $48.3 \pm 11.3^{+++}$ & $40.7 \pm 14.7$ & $48.0 \pm 11.5^{\star t}$ \\
\hline$B M I\left(\mathrm{~kg} / \mathrm{m}^{2}\right)$ & $25.2 \pm 3.2$ & $29.2 \pm 3.3 * * *$ & $25.5 \pm 3.09$ & $29.5 \pm 3.62^{+++}$ & $25.1 \pm 3.1$ & $29.2 \pm 3.4^{+*}$ \\
\hline Waist (cm) & $88.1 \pm 9.3$ & $101.2 \pm 7.7 * * *$ & $89.5 \pm 8.9$ & $101.8 \pm 9.1^{+++}$ & $88.0 \pm 8.9$ & $101.1 \pm 8.3^{\star t}$ \\
\hline$S B P(m m H g)$ & $127.4 \pm 15.2$ & $133.2 \pm 13.8^{*}$ & $126.8 \pm 14.6$ & $135.6 \pm 13.6^{+++}$ & $127.4 \pm 15.2$ & $133.2 \pm 13.8 \mathrm{tt}$ \\
\hline $\mathrm{DBP}(\mathrm{mm} \mathrm{Hg})$ & $78.9 \pm 8.8$ & $82.8 \pm 7.1 * *$ & $78.9 \pm 8.5$ & $83.8 \pm 6.9^{+++}$ & $78.9 \pm 8.8$ & $82.8 \pm 7.1^{+*}$ \\
\hline Hypertension (n/\%) & $53 / 33.1$ & $74 / 54.8 * *$ & $55 / 30.0$ & $72 / 64.8^{+++}$ & $41 / 25.9$ & $86 / 62.7^{+1}$ \\
\hline Smoking (n/\%) & $28 / 17.5$ & $47 / 34.8 * * *$ & $33 / 17.9$ & $41 / 36.9^{+++}$ & $29 / 18.3$ & $46 / 33.5^{\mathrm{t}}$ \\
\hline$T C(\mathrm{mmol} / \mathrm{l})$ & $6.48 \pm 1.88$ & $6.86 \pm 1.77$ & $6.53 \pm 1.81$ & $6.85 \pm 1.88$ & $6.53 \pm 1.90$ & $6.79 \pm 1.76$ \\
\hline$T G(\mathrm{mmol} / \mathrm{l})$ & $\begin{array}{l}2.09 \pm 2.55 \\
(1.59)\end{array}$ & $\begin{array}{l}5.30 \pm 4.98 * * * \\
(3.68)\end{array}$ & $\begin{array}{l}2.48 \pm 2.66 \\
(1.75)\end{array}$ & $\begin{array}{l}5.29 \pm 5.40^{+++} \\
(3.61)\end{array}$ & $\begin{array}{l}2.30 \pm 2.74 \\
(1.63)\end{array}$ & $\begin{array}{l}5.02 \pm 4.99+t \\
(3.46)\end{array}$ \\
\hline$A I P: \log (T G / H D L-C)$ & $0.09 \pm 0.30$ & $0.60 \pm 0.29 * * *$ & $0.15 \pm 0.33$ & $0.59 \pm 0.33^{+++}$ & $0.10 \pm 0.32$ & $0.57 \pm 0.31^{\star *}$ \\
\hline NonHDL-C (mmol/l) & $5.10 \pm 1.90$ & $5.78 \pm 1.77 * *$ & $5.17 \pm 1.84$ & $5.81 \pm 1.87^{++}$ & $5.13 \pm 1.93$ & $5.74 \pm 1.75^{\star *}$ \\
\hline$H D L-C(\mathrm{mmol} / \mathrm{l})$ & $1.38 \pm 0.35$ & $1.07 \pm 0.26^{* * *}$ & $1.36 \pm 0.32$ & $1.04 \pm 0.29^{+++}$ & $1.40 \pm 0.34$ & $1.05 \pm 0.24^{\star *}$ \\
\hline$L D L-C(\mathrm{mmol} / \mathrm{l})$ & $4.20 \pm 1.36$ & $3.95 \pm 1.44$ & $4.14 \pm 1.33$ & $4.01 \pm 1.54$ & $4.20 \pm 1.37$ & $3.96 \pm 1.43$ \\
\hline Apo A-1 $(g / l)$ & $1.47 \pm 0.26$ & $1.35 \pm 0.25 * * *$ & $1.46 \pm 0.26$ & $1.32 \pm 0.24^{+++}$ & $1.48 \pm 0.27$ & $1.33 \pm 0.23^{+t}$ \\
\hline Apo B $(g / l)$ & $1.21 \pm 0.31$ & $1.25 \pm 0.34$ & $1.21 \pm 0.31$ & $1.25 \pm 0.35$ & $1.20 \pm 0.31$ & $1.25 \pm 0.33$ \\
\hline Аро В/Apo A-1 & $0.86 \pm 0.32$ & $0.96 \pm 0.41^{*}$ & $0.86 \pm 0.32$ & $0.98 \pm 0.43^{++}$ & $0.84 \pm 0.33$ & $0.97 \pm 0.40^{*}$ \\
\hline $\operatorname{Lp}(a)(g / l)$ & $\begin{array}{l}0.42 \pm 0.45 \\
(0.24)\end{array}$ & $\begin{array}{l}0.28 \pm 0.36^{* *} \\
(0.13)\end{array}$ & $\begin{array}{l}0.41 \pm 0.42 \\
(0.24)\end{array}$ & $\begin{array}{l}0.27 \pm 0.39^{++} \\
(0.12)\end{array}$ & $\begin{array}{l}0.41 \pm 0.43 \\
(0.23)\end{array}$ & $\begin{array}{l}0.29 \pm 0.39 \$+ \\
(0.13)\end{array}$ \\
\hline Fibrinogen $(g / l)$ & $3.05 \pm 0.71$ & $3.2 \pm 0.7 * *$ & $3.08 \pm 0.72$ & $3.19 \pm 0.71^{++}$ & $3.0 \pm 0.73$ & $3.19 \pm 0.69^{*}$ \\
\hline$v W F(\%)$ & $132 \pm 50$ & $126 \pm 50$ & $129 \pm 51$ & $128 \pm 48$ & $131 \pm 53$ & $127 \pm 47$ \\
\hline$t-P A(n g / m l)$ & $\begin{array}{l}4.26 \pm 4.33 \\
(3.00)\end{array}$ & $\begin{array}{l}4.94 \pm 4.50 \\
(3.05)\end{array}$ & $\begin{array}{l}4.10 \pm 4.28 \\
(2.90)\end{array}$ & $\begin{array}{l}5.27 \pm 4.49^{++} \\
(3.40)\end{array}$ & $\begin{array}{l}4.24 \pm 4.38 \\
(3.00)\end{array}$ & $\begin{array}{l}4.95 \pm 4.44 \\
(3.00)\end{array}$ \\
\hline$P A I-1(\mathrm{ng} / \mathrm{ml})$ & $\begin{array}{l}77 \pm 39 \\
(74)\end{array}$ & $\begin{array}{l}94 \pm 44 * * * \\
(93)\end{array}$ & $\begin{array}{l}78 \pm 37 \\
(76)\end{array}$ & $\begin{array}{l}97 \pm 47^{++} \\
(93)\end{array}$ & $\begin{array}{l}76 \pm 37 \\
(74)\end{array}$ & $\begin{array}{l}96 \pm 46^{+4} \\
(92)\end{array}$ \\
\hline$h s C R P(m g / l)$ & $\begin{array}{l}1.66 \pm 2.02 \\
(1.04)\end{array}$ & $\begin{array}{l}2.76 \pm 3.17 * * * \\
(1.79)\end{array}$ & $\begin{array}{l}1.69 \pm 2.13 \\
(1.06)\end{array}$ & $\begin{array}{l}2.98 \pm 3.24^{+++} \\
(2.05)\end{array}$ & $\begin{array}{l}1.54 \pm 1.89 \\
(1.00)\end{array}$ & $\begin{array}{l}2.88 \pm 3.20^{4 *} \\
(1.90)\end{array}$ \\
\hline Glucose (mmol/l) & $5.11 \pm 0.68$ & $5.39 \pm 0.79 *$ & $5.03 \pm 0.60$ & $5.57 \pm 0.80^{+++}$ & $5.02 \pm 0.62$ & $5.49 \pm 0.80^{*+*}$ \\
\hline Insulin (mIU/l) & $\begin{array}{l}7.8 \pm 4.9 \\
(6.8)\end{array}$ & $\begin{array}{l}11.8 \pm 6.9 * * * \\
(10.9)\end{array}$ & $\begin{array}{l}7.7 \pm 4.78 \\
(6.8)\end{array}$ & $\begin{array}{l}12.8 \pm 7.2^{+++} \\
(11.2)\end{array}$ & $\begin{array}{l}7.6 \pm 4.9 \\
(6.5)\end{array}$ & $\begin{array}{l}12.0 \pm 6.8^{\dagger t} \\
(11.0)\end{array}$ \\
\hline$H O M A$ & $\begin{array}{l}1.8 \pm 1.2 \\
(1.5)\end{array}$ & $\begin{array}{l}2.8 \pm 1.9 * * * \\
(2.5)\end{array}$ & $\begin{array}{l}1.7 \pm 1.1 \\
(1.5)\end{array}$ & $\begin{array}{l}3.2 \pm 1.9^{+++} \\
(2.7)\end{array}$ & $\begin{array}{l}1.7 \pm 1.2 \\
(1.4)\end{array}$ & $\begin{array}{l}2.9 \pm 1.8^{t+t} \\
(2.6)\end{array}$ \\
\hline C peptide $(\mathrm{mg} / \mathrm{l})$ & $\begin{array}{l}2.22 \pm 1.21 \\
(2.0)\end{array}$ & $\begin{array}{l}3.15 \pm 1.44 * * * \\
(2.98)\end{array}$ & $\begin{array}{l}2.21 \pm 1.14 \\
(2.04)\end{array}$ & $\begin{array}{l}3.37 \pm 1.49^{+++} \\
(3.19)\end{array}$ & $\begin{array}{l}2.1 \pm 1.1 \\
(1.9)\end{array}$ & $\begin{array}{l}3.2 \pm 1.4^{\star *} \\
(3.0)\end{array}$ \\
\hline Proinsulin (mIU/l) & $\begin{array}{l}12.5 \pm 7.8 \\
(10.4)\end{array}$ & $\begin{array}{l}18.5 \pm 11.0 * * * \\
(15.4)\end{array}$ & $\begin{array}{l}12.6 \pm 8.0 \\
(10.5)\end{array}$ & $\begin{array}{l}19.9 \pm 11.1^{+++} \\
(15.7)\end{array}$ & $\begin{array}{l}12.5 \pm 8.4 \\
(10.3)\end{array}$ & $\begin{array}{l}18.5 \pm 10.5^{*+4} \\
(15.5)\end{array}$ \\
\hline Adiponection $(\mathrm{mg} / \mathrm{l})$ & $\begin{array}{l}9.6 \pm 5.4 \\
(8.7)\end{array}$ & $\begin{array}{l}7.4 \pm 4.5^{* * * *} \\
(6.5)\end{array}$ & $\begin{array}{l}9.4 \pm 5.1 \\
(8.4)\end{array}$ & $\begin{array}{l}7.2 \pm 4.8^{+++} \\
(5.9)\end{array}$ & $\begin{array}{l}9.5 \pm 5.2 \\
(8.5)\end{array}$ & $\begin{array}{l}7.4 \pm 4.7^{+1+t} \\
(6.2)\end{array}$ \\
\hline$I M T(\mathrm{~mm}) \dagger$ & $0.66 \pm 0.14$ & $0.71 \pm 0.14$ & $0.66 \pm 0.13$ & $0.73 \pm 0.15$ & $0.65 \pm 0.13$ & $0.72 \pm 0.14$ \\
\hline
\end{tabular}

Hypertriglyceridemic waist (HTGW) in men is defined as waist $\geq 90 \mathrm{~cm}$ and TG $\geq 2.0 \mathrm{mmol} / \mathrm{l} ; \mathrm{MS}$ - harmonized definition of metabolic syndrome (Alberti et al. 2009); MS-I: WC $\geq 102 \mathrm{~cm}$ in men and $\geq 88 \mathrm{~cm}$ in women; MS-II: WC $\geq 94 \mathrm{~cm}$ in men and $\geq 80 \mathrm{~cm}$ in women. Data are expressed as mean \pm standard deviation and for variables with non-normal distribution also as (median). Statistical significance (after adjustment for age) of difference between subjects with and without HTGW: $* p<0.05, * * p<0.01, * * * p<0.001$; with and without MS-I: ${ }^{+} p<0.05,{ }^{++} p<0.01,{ }^{++} p<0.001$; with and without MS-II: ${ }^{\ddagger} p<0.05,{ }^{\neq \neq} p<0.01,{ }^{, \neq \neq} p<0.001$; +IMT was assessed in a subset of 173 men. 
Table 2. Physical characteristics and cardiometabolic risk profile of FEMALE dyslipidemic subjects with (1) and without (0) HTG W, MS-I and MS-II.

\begin{tabular}{|c|c|c|c|c|c|c|}
\hline Parameter & $\begin{array}{c}\text { HTGW (0) } \\
n=211\end{array}$ & $\begin{array}{c}\text { HTGW (1) } \\
n=101\end{array}$ & $\begin{array}{c}\text { MS-I (0) } \\
\text { n=224 }\end{array}$ & $\begin{array}{c}\text { MS-I (1) } \\
\mathbf{n}=\mathbf{8 8}\end{array}$ & $\begin{array}{c}\text { MS-II (0) } \\
\mathbf{n}=\mathbf{2 0 7}\end{array}$ & $\begin{array}{c}\text { MS-II (1) } \\
n=105\end{array}$ \\
\hline Age (years) & $43.9 \pm 14.4$ & $53.6 \pm 11.1^{* * *}$ & $44.4 \pm 14.7$ & $53.9 \pm 9.7^{+++}$ & $43.3 \pm 14.6$ & $54.5 \pm 9.7^{+*}$ \\
\hline$B M I\left(\mathrm{~kg} / \mathrm{m}^{2}\right)$ & $23.9 \pm 3.1$ & $29.1 \pm 3.6^{* * *}$ & $24.0 \pm 3.0$ & $29.5 \pm 3.8^{+++}$ & $23.9 \pm 3.0$ & $28.8 \pm 3.9^{+1}$ \\
\hline Waist (cm) & $77.2 \pm 8.9$ & $94.2 \pm 7.4^{* * *}$ & $78.2 \pm 9.3$ & $94.2 \pm 8.8^{+++}$ & $77.7 \pm 9.3$ & $92.7 \pm 8.9^{+1 *}$ \\
\hline$S B P(m m H g)$ & $124.7 \pm 14.3$ & $136.9 \pm 16.9 * * *$ & $124.0 \pm 13.8$ & $140.5 \pm 15.9^{+++}$ & $123.5 \pm 13.4$ & $138.8 \pm 16.4^{4+4}$ \\
\hline$D B P(m m ~ H g)$ & $77.1 \pm 8.3$ & $82.2 \pm 9.3 * *$ & $76.7 \pm 8.1$ & $84.1 \pm 8.9^{+++}$ & $76.5 \pm 8.2$ & $83.2 \pm 8.7^{+1}$ \\
\hline Hypertension (n/\%) & $45 / 21.3$ & $59 / 58.4 * * *$ & $43 / 19.1$ & $61 / 69.3^{+++}$ & $36 / 17.3$ & $68 / 64.7^{*+4}$ \\
\hline Smoking (n/\%) & $33 / 15.4$ & $21 / 20.7$ & $32 / 14.3$ & $22 / 25.0^{+}$ & $29 / 14.0$ & $25 / 23.8^{\ddagger}$ \\
\hline$T C(\mathrm{mmol} / \mathrm{l})$ & $6.55 \pm 1.16$ & $7.32 \pm 1.78^{* * *}$ & $6.63 \pm 1.26$ & $7.27 \pm 1.7^{++}$ & $6.5 \pm 1.18$ & $7.28 \pm 1.75^{\star}$ \\
\hline$T G(\mathrm{mmol} / \mathrm{l})$ & $\begin{array}{l}1.50 \pm 0.88 \\
(1.37)\end{array}$ & $\begin{array}{l}3.59 \pm 3.96 * * * \\
(2.59)\end{array}$ & $\begin{array}{l}1.61 \pm 1.45 \\
(1.4)\end{array}$ & $\begin{array}{l}3.61 \pm 3.8^{+++} \\
(2.67)\end{array}$ & $\begin{array}{l}1.45 \pm 0.63 \\
(1.36)\end{array}$ & $\begin{array}{l}3.62 \pm 3.95^{+t} \\
(2.59)\end{array}$ \\
\hline$A I P: \log (T G / H D L-C)$ & $-0.10 \pm 0.25$ & $0.33 \pm 0.27 * * *$ & $-0.08 \pm 0.26$ & $0.35 \pm 0.28^{+++}$ & $-0.11 \pm 0.23$ & $0.34 \pm 0.28^{\star t+}$ \\
\hline NonHDL-C (mmol/l) & $4.79 \pm 1.18$ & $5.95 \pm 1.72 * * *$ & $4.88 \pm 1.29$ & $5.93 \pm 1.64^{+++}$ & $4.79 \pm 1.20$ & $5.92 \pm 1.68^{\mathrm{tt}}$ \\
\hline$H D L-C(\mathrm{mmol} / \mathrm{l})$ & $1.75 \pm 0.44$ & $1.37 \pm 0.34 * * *$ & $1.74 \pm 0.42$ & $1.34 \pm 0.38^{+++}$ & $1.77 \pm 0.42$ & $1.36 \pm 0.35^{\star t}$ \\
\hline$L D L-C(\mathrm{mmol} / \mathrm{l})$ & $4.10 \pm 1.07$ & $4.53 \pm 1.37$ & $4.14 \pm 1.16$ & $4.50 \pm 1.21$ & $4.10 \pm 1.11$ & $4.51 \pm 1.30$ \\
\hline Apo A-1 $(\mathrm{g} / \mathrm{l})$ & $1.79 \pm 0.34$ & $1.58 \pm 0.27 * * *$ & $1.79 \pm 0.33$ & $1.55 \pm 0.28^{+++}$ & $1.80 \pm 0.33$ & $1.56 \pm 0.27^{+t}$ \\
\hline Apo B $(g / l)$ & $1.18 \pm 027$ & $1.37 \pm 0.36^{* * *}$ & $1.19 \pm 0.28$ & $1.37 \pm 0.35^{+++}$ & $1.17 \pm 0.27$ & $1.37 \pm 0.35^{*+}$ \\
\hline Apo B/Apo A-1 & $0.68 \pm 0.22$ & $0.89 \pm 0.29 * * *$ & $0.69 \pm 0.22$ & $0.90 \pm 0.29^{+++}$ & $0.68 \pm 0.21$ & $0.89 \pm 0.29$ \\
\hline $\operatorname{Lp}(a)(g / l)$ & $\begin{array}{l}0.44 \pm 0.52 \\
(0.21)\end{array}$ & $\begin{array}{l}0.30 \pm 0.33 * * * \\
(0.16)\end{array}$ & $\begin{array}{l}0.44 \pm 0.51 \\
(0.23)\end{array}$ & $\begin{array}{l}0.29 \pm 0.34^{+++} \\
(0.13)\end{array}$ & $\begin{array}{l}0.43 \pm 0.52 \\
(0.22)\end{array}$ & $\begin{array}{l}0.32 \pm 0.36^{\star *} \\
(0.16)\end{array}$ \\
\hline Fibrinogen $(g / l)$ & $3.2 \pm 0.75$ & $3.4 \pm 0.71 *$ & $3.19 \pm 0.73$ & $3.48 \pm 0.74^{+}$ & $3.17 \pm 0.73$ & $3.46 \pm 0.73^{*}$ \\
\hline$v W F(\%)$ & $128 \pm 45$ & $142 \pm 58$ & $130 \pm 46$ & $140 \pm 59$ & $128 \pm 45$ & $143 \pm 58$ \\
\hline$t-P A(n g / m l)$ & $\begin{array}{l}3.19 \pm 3.36 \\
(2.1)\end{array}$ & $\begin{array}{l}4.02 \pm 3.6 \\
(3.0)\end{array}$ & $\begin{array}{l}2.97 \pm 2.78 \\
(2.1)\end{array}$ & $\begin{array}{l}4.7 \pm 4.57^{++} \\
(3.0)\end{array}$ & $\begin{array}{l}2.83 \pm 2.68 \\
(2.0)\end{array}$ & $\begin{array}{l}4.72 \pm 4.40^{+1} \\
(3.0)\end{array}$ \\
\hline PAI-1 (ng/ml) & $\begin{array}{l}59 \pm 33 \\
(53)\end{array}$ & $\begin{array}{l}78 \pm 41^{* * * *} \\
(76)\end{array}$ & $\begin{array}{l}59 \pm 32 \\
(56)\end{array}$ & $\begin{array}{l}82 \pm 43^{+++} \\
(79)\end{array}$ & $\begin{array}{l}58 \pm 32 \\
(52)\end{array}$ & $\begin{array}{l}80 \pm 41^{+1} \\
(78)\end{array}$ \\
\hline$h s C R P(m g / l)$ & $\begin{array}{l}2.63 \pm 3.47 \\
(1.4)\end{array}$ & $\begin{array}{l}3.14 \pm 3.43^{*} \\
(2.15)\end{array}$ & $\begin{array}{l}2.66 \pm 3.5 \\
(1.32)\end{array}$ & $\begin{array}{l}3.13 \pm 3.36^{+} \\
(2.22)\end{array}$ & $\begin{array}{l}2.59 \pm 3.5 \\
(1.31)\end{array}$ & $\begin{array}{l}3.18 \pm 3.36^{\star} \\
(2.22)\end{array}$ \\
\hline Glucose (mmol/l) & $4.82 \pm 0.61$ & $5.44 \pm 1.17 * * *$ & $4.79 \pm 0.55$ & $5.61 \pm 1.23^{+++}$ & $4.78 \pm 0.53$ & $5.50 \pm 1.19^{*+t}$ \\
\hline Insulin (mIU/l) & $\begin{array}{l}7.3 \pm 3.9 \\
(6.9)\end{array}$ & $\begin{array}{l}11.0 \pm 5.5 * * * \\
(10.3)\end{array}$ & $\begin{array}{l}7.2 \pm 3.6 \\
(6.9)\end{array}$ & $\begin{array}{l}11.7 \pm 6.0^{+++} \\
(10.7)\end{array}$ & $\begin{array}{l}7.1 \pm 3.6 \\
(6.9)\end{array}$ & $\begin{array}{l}11.2 \pm 5.7^{*+4} \\
(11.2)\end{array}$ \\
\hline HOMA & $\begin{array}{l}1.5 \pm 0.9 \\
(1.4)\end{array}$ & $\begin{array}{l}2.6 \pm 1.5 * * * \\
(2.2)\end{array}$ & $\begin{array}{l}1.5 \pm 0.8 \\
(1.4)\end{array}$ & $\begin{array}{l}2.9 \pm 1.6^{+++} \\
(2.6)\end{array}$ & $\begin{array}{l}1.5 \pm 0.8 \\
(1.4)\end{array}$ & $\begin{array}{l}2.7 \pm 1.5^{+1} \\
(2.4)\end{array}$ \\
\hline$C$ peptide $(\mathrm{mg} / \mathrm{l})$ & $\begin{array}{l}2.03 \pm 0.81 \\
(1.94)\end{array}$ & $\begin{array}{l}3.16 \pm 1.26^{* * * *} \\
(2.92)\end{array}$ & $\begin{array}{l}2.03 \pm 0.78 \\
(1.95)\end{array}$ & $\begin{array}{l}3.33 \pm 1.28^{+++} \\
(3.10)\end{array}$ & $\begin{array}{l}1.98 \pm 0.76 \\
(1.91)\end{array}$ & $\begin{array}{l}3.21 \pm 1.24^{+t} \\
(3.02)\end{array}$ \\
\hline Proinsulin (mIU/l) & $\begin{array}{l}10.7 \pm 4.9 \\
(9.7)\end{array}$ & $\begin{array}{l}16.4 \pm 10.1^{* * *} \\
(13.2)\end{array}$ & $\begin{array}{l}10.6 \pm 4.8 \\
(9.6)\end{array}$ & $\begin{array}{l}18.0 \pm 10.4^{+++} \\
(15.4)\end{array}$ & $\begin{array}{l}10.5 \pm 4.9 \\
(9.5)\end{array}$ & $\begin{array}{l}16.9 \pm 9.9^{\dagger+} \\
(14.7)\end{array}$ \\
\hline Adiponection $(\mathrm{mg} / \mathrm{l})$ & $\begin{array}{l}15.2 \pm 10.1 \\
(13.0)\end{array}$ & $\begin{array}{l}12.5 \pm 7.6^{* *} \\
(10.5)\end{array}$ & $\begin{array}{l}15.1 \pm 10.1 \\
(12.9)\end{array}$ & $\begin{array}{l}12.4 \pm 7.2^{++} \\
(10.3)\end{array}$ & $\begin{array}{l}15.3 \pm 10.5 \\
(12.9)\end{array}$ & $\begin{array}{l}12.4 \pm 6.8^{4} \\
(11.2)\end{array}$ \\
\hline$I M T(m m) \dagger$ & $0.64 \pm 0.12$ & $0.74 \pm 0.12 *$ & $0.64 \pm 0.12$ & $0.74 \pm 0.11$ & $0.64 \pm 0.12$ & $0.73 \pm 0.11$ \\
\hline
\end{tabular}

Hypertriglyceridemic waist (HTGW) in females is defined as waist $\geq 85 \mathrm{~cm}$ and TG $\geq 1.5 \mathrm{mmol} / \mathrm{l}$; MS - harmonized definition of metabolic syndrome (Alberti et al. 2009); MS-I: WC $\geq 102 \mathrm{~cm}$ in men and $\geq 88 \mathrm{~cm}$ in women; MS-II: WC $\geq 94 \mathrm{~cm}$ in men and $\geq 80 \mathrm{~cm}$ in women. Data are expressed as mean \pm standard deviation and for variables with non-normal distribution also as (median). Statistical significance (after adjustment for age) of difference between subjects with and without HTGW: $* p<0.05, * * p<0.01, * * * p<0.001$; with and without MS-I: ${ }^{+} p<0.05,{ }^{++} p<0.01,{ }^{++} p<0.001$; with and without MS-II: ${ }^{\ddagger} p<0.05,{ }^{\ddagger \ddagger} p<0.01,{ }^{\ddagger \neq \ddagger} p<0.001 ;+$ IMT was assessed in a subset of 195 women. 
Both the men and women with the HTGW phenotype were characterized by a more atherogenic lipid profile, increased markers of insulin resistance, and a proinflammatory and prothrombotic state in comparison with those without HTGW. Despite the fact that blood pressure does not belong among the diagnostic criteria of HTGW, both the men and women with HTGW had a significantly higher prevalence of hypertension and higher SBP and DBP. Significantly more smokers were found among the male HTGW subjects.

Increased IMT reached statistical significance after adjustment for age only in the women with HTGW $(p=0.024)$ but after further adjustment for other risk factors included in the SCORE risk estimation system (TC, smoking, SBP), the effect of HTGW on IMT was no longer significant. Increased IMT in the HTGW men and in MS-I and MS-II in both genders did not reach statistical significance after adjustment for age alone.

In our study, the prevalence of the HTGW phenotype, MS-I, and MS-II was $45.7 \%, 37.6 \%$, and $46.4 \%$, respectively in the men and $32.3 \%, 28.2 \%$, and $33.6 \%$, respectively in the women.

$69 \%$ of the men and $77.2 \%$ of the women with HTGW phenotype also met MS-I criteria and $85.1 \%$ of the men and $86.1 \%$ of the women also met the MS-II criteria.

Markers of insulin resistance and all the cardiometabolic risk factors that were followed up were expressed most in those who fulfilled the criteria for both HTGW and MS-I ( $\mathrm{n}=171)$ in comparison with those with HTGW alone $(n=65)$ and MS-I alone $(n=25)$. Nevertheless, the members of the group with HTGW alone did not differ significantly from those with MS-I alone, with the exception of lower SBP and DBP in the subjects with HTGW alone.

MS-II was slightly more prevalent than HTGW. The criteria for MS-II + HTGW, MS-II alone, and HTGW alone were fulfilled by 202, 41, and 35 subjects respectively.

\section{Discussion}

In agreement with other studies, our subjects with HTGW had an atherogenic lipid profile (Arsenault et al. 2010, Blackburn et al. 2009, 2012) with increased triglycerides, non-HDL-C, AIP, ApoB/ApoA1, and low HDL-C and ApoA1, but without significant changes in LDL-C (Blackburn et al. 2009, 2012). This shows that in these subjects we cannot rely only on LDL-C, which is considered the main target of hypolipidemic therapy, but we should also consider non-HDL-C or Apo B.

Surprisingly, we found lower levels of $\mathrm{Lp}$ (a) in all three cardiometabolic risk phenotypes (HTGW, MS-I and MS-II) in both the men and women - a finding which had not previously been described. This finding needs further investigation.

Contrary to the men, our female subjects with HTGW also had significantly higher levels of TC and Apo B. This might show that abdominal obesity with hypertriglyceridemia is even more atherogenic in women than in men. Also in agreement with previous studies are the findings of increased markers of insulin resistance (Blackburn et al. 2009, Han et al. 2014), increased markers of inflammation (Arsenault et al. 2010, Blackburn et al. 2009), and lower levels of adiponectin (Blackburn et al. 2009) in our subjects with HTGW. We have not found an evaluation of prothrombotic markers in subjects with HTGW in the literature but it is well known that visceral obesity and MS are also associated with prothrombotic changes (Pinheiro Volp et al. 2015, Festa et al. 1999, Palomo et al. 2009, Sakkinen et al. 2000). In the study of Festa et al. (1999), the decrease in insulin sensitivity was an independent factor associated with high fibrinogen and PAI-1 concentrations.

In our study, significant differences in all the parameters that were followed up were very similar in all three phenotypes associated with cardiometabolic risk (HTGW, MS-I, and MS-II).

In the whole group of men and women, HTGW was diagnosed more frequently than MS-I (n: 236 vs. 199). On the contrary, MS-II was only slightly more prevalent than HTGW (n: 242 vs. 236). It is possible that the cutoff value of the waist circumference $(\geq 102 \mathrm{~cm}$ in men and $\geq 88 \mathrm{~cm}$ in women) in MS-I may be too high and may therefore lead to the misclassification of many subjects with cardiometabolic risk.

Blackburn et al. (2009) compared the HTGW phenotype with MS defined by the National Cholesterol Education Program - Adult Treatment Panel III (NCEP ATP III 2002) and the clinical criteria of the International Diabetes Federation (IDF) (Alberti et al. 2005), with similar results.

IMT is an established non-invasive method for the detection of early atherosclerotic disease (Bots et al. 1997). Longitudinal studies have shown a strong association between IMT and a future risk of myocardial infarction and stroke (Bots et al. 1997, O'Leary et al. 1999). In our study, only the women with HTGW showed 
increased IMT after adjustment for age but after further adjustment for factors included in SCORE (TC, SBP, smoking) it was no longer significant.

The association of HTGW with IMT was also evaluated in a multiethnic general population (Gasevic et al. 2014). These authors found increased IMT in both men and women with HTGW but similarly as in our study, after further adjustment for traditional risk factors for atherosclerosis, the effect of HTGW was no longer significant.

Tankó et al. (2005) compared an enlarged waist $\geq 88 \mathrm{~cm}$ combined with elevated triglycerides $\geq 1.45 \mathrm{mmol} / 1$ (EWET) with MS-NCEP. In their prospective study, EWET was associated with a 4.7 -fold increased risk and MS-NCEP with a 3.2-fold increased risk of fatal cardiovascular events $(\mathrm{p}<0.001$ for both). Their subjects with EWET did not differ from MS in terms of their waist and lipid parameters but had significantly lower systolic and diastolic blood pressure. Nevertheless, those who had EWET alone at the baseline had a higher progression rate of aortic calcification compared with those who had MS-NCEP alone $(\mathrm{p}<0.05)$. After analysis of the association of individual components of EWET and MS-NCEP with cardiovascular mortality, they came to the conclusion that the combined presence of an enlarged waist and elevated triglycerides may be the best indicator of cardiovascular risk in postmenopausal women. Other components of MS-NCEP add little medical value (Tankó et al. 2005).

A combination of an enlarged waist and elevated triglycerides has an advantage in indentifying individuals with atherogenic "lipid over-accumulation". As waist circumference cannot fully discriminate visceral adiposity from subcutaneous abdominal obesity, elevated triglyceride levels have been adopted as a marker of dysfunctional visceral adipose tissue (Lemieux et al. 2000). It was recently suggested that a limited ability of subcutaneous fat to store excess energy results in an overflow of triglycerides to intraabdominal (visceral) fat and to ectopic sites, such as the liver, the epicardial fat, and the skeletal muscle. This leads to metabolic dysfunction of these organs and the development of dyslipidemia and insulin resistance (Despres 2012). The expansion of adipose tissue leads to adipocyte hypertrophy, adipocyte hypoxia and the activation of oxidative and inflammatory cellular stress pathways with the activation of production of cytokines and other proinflammatory signals. Locally produced chemokines attract pro-inflammatory macrophages to adipose tissue, which form a crown shape structure around the dead and/or sick big adipocytes (Heilbronn and Campbell 2008). With advanced expansion of adipose tissue, M2 antiinflammatory macrophages acquire an M1 proinflammatory phenotype. Proinflammatory cytokines including TNF- $\alpha$, IL- 6 , IL-1 $\beta$ produced by M1 further exacerbate local inflammation, promoting insulin resistance (Heilbronn and Campbell 2008, Finucane et al. 2012). Dysbalance in the production of specific proteins and hormones produced by omental and mesenteric adipose tissue, such as inflammatory adipokines (resistin, leptin, adiponectin), angiotensinogen, and cortisol can also contribute to cardiometabolic disease (Klein et al. 2007). Factors associated with a preferential accumulation of visceral fat and with features of insulin resistance include, among others, genetic predisposition, maladaptive response to stress, and smoking (Despres 2012). The higher prevalence of smokers in all three phenotypes associated with cardiometabolic risk (HTGW, MS-I, and MS-II) in our study supports the importance of the role of smoking in the development of visceral obesity, insulin resistance, and a proatherogenic lipid profile. It is also in agreement with our previvous study (Cibičkova et al. 2014).

Our results demonstrate that HTGW is associated with a proatherogenic lipid profile, elevated blood pressure, the presence of insulin resistance, and proinflammatory and prothrombotic changes with impaired fibrinolysis, similarly to individuals meeting the harmonized definition of MS. Thus it is not surprising that in multiple prospective studies HTGW has been shown to be associated with an increased risk of developing cardiovascular disease (Czernikow et al. 2007, Tanko et al. 2005, Arsenault et al. 2010, St-Pierre et al. 2007) and type 2 diabetes mellitus (Zhang et al. 2012, Carlsson et al. 2013, Han et al. 2014, He et al. 2013, Díaz-Santana et al. 2014).

Thus, HTGW represents a simple screening phenotype for the detection of subjects with cardiometabolic risk. Plasma triglyceride levels are available from any standard lipid profile and waist circumference can be measured at no cost. Nevertheless, despite the increasing prevalence of obesity and especially abdominal obesity, the measurement of waist circumference in daily clinical practice is still very rare. Gupta et al. (2012) surveyed Canadian primary care physicians and found that WC was routinely measured by only $6 \%$ of these physicians. 


\section{Conclusion}

The inclusion of HTGW as a simple, easily accessible, and inexpensive screening tool into daily clinical practice in primary care could lead to the detection of large numbers of subjects with cardiometabolic risk. It is especially important in this era of the increasing prevalence of obesity and type 2 diabetes mellitus, which is unquestionably a predictor of a further epidemic of cardiovascular disease.

\section{Conflict of Interest}

There is no conflict of interest.

\section{Acknowledgements}

Supported by grant Nr. LF $-2015-015$.

\section{References}

ALBERTI KG, ZIMMET P, SHAW J: The metabolic syndrome - a new world wide definition. Lancet 366: 1059-1062, 2005.

ALBERTI KG, ECKEL RH, GRUNDY SM, ZIMMET PZ, CLEEMAN JI, DONATO KA, FRUCHART JC, JAMES WP, LORIA CM, SMITH SC JR: Harmonizing the metabolic syndrome: a joint interim statement of the International Diabetes Federation Task Force on Epidemiology and Prevention; National Heart, Lung, and Blood Institute; American Heart Association; World Heart Federation; International Atherosclerosis Society; and International Association for the Study of Obesity. Circulation 120: 1640-1645, 2009.

ARSENAULT BJ, LEMIEUX I, DESPRÉS JP, WAREHAM NJ, KASTELEIN JJP, KHAW KT, BOEKHOLT SM: The hypertriglyceridemic-waist phenotype and the risk of coronary artery disease: results from the EPICNorfolk Prospective Population Study. CMAJ 182: 1427-1432, 2010.

BLACKBURN P, LEMIEUX I, ALMÉRAS N, BERGERON J, CÔTÉ M, TREMBLAY A, LAMARCHE B, DESPRÉS JP: The hypertriglyceridemic waist phenotype versus the National Cholesterol Education ProgramAdult Treatment Panel III and International Diabetes Federation clinical criteria to identify high-risk men with an altered cardiometabolic risk profile. Metabolism 58: 1123-1130, 2009.

BLACKBURN P, LEMIEUX I, LAMARCHE B, BERGERON J, PERRON P, TREMBLAY G, GAUDET D, DESPRÉS JP: Hypertriglyceridemic waist: a simple clinical phenotype associated with coronary artery disease in women. Metabolism 61: 56-64, 2012.

BOTS ML, HOES AW, KOUDSTAAL PJ, HOFMAN A, GROBBEE DE: Common karotid intima-media thickness and risk of stroke and myocardial infarction: the Rotterdam Study. Circulation 96: 1432-1437, 1997.

BRUTHANS J, CÍFKOVÁ R, LÁNSKÁ V, O'FLAHERTY M, CRITCHLEY JA, HOLUB J, JANSKÝ P, ZVÁROVÁ J, CAPEWELL S: Explaining the decline in coronary heart disease mortality in the Czech Republic between 1985 and 2007. Eur J Prev Cardiol 21: 829-839, 2014.

CARLSSON AC, RISÉRUS U, ÄRNLÖV J: Hypertriglyceridemic waist phenotype is associated with decreased insulin sensitivity and incident diabetes in elderly men. Obesity 22: 526-529, 2014.

CIBIČKOVÁ L', KARÁSEK D, LANGOVÁ K, VAVERKOVÁ H, ORSÁG J, LUKEŠ J, NOVOTNÝ D: Correlation of lipid parameters and markers of insulin resistance: does smoking make a difference? Physiol Res $\mathbf{6 3}$ (Suppl 3): S387-S393, 2014.

CZERNICHOW S, BRUCKERT E, BERTRAIS S, GALAN P, HERCBERG S, OPPERT JM: Hypertriglyceridemic waist and 7.5-year prospective risk of cardiovascular disease in asymptomatic middle-aged men. Int J Obes (Lond) 31: 791-796, 2007.

DESPRÉS JP: Body fat distribution and risk of cardiovascular disease: an update. Circulation 126: 1301-1313, 2012.

DÍAZ-SANTANA MV, SUÁREZ PÉREZ EL, ORTIZ MARTÍNEZ AP, GUZMÁN SERRANO M, PÉREZ CARDONA CM: Association between the hypertriglyceridemic waist phenotype, prediabetes, and diabetes mellitus among adults in Puerto Rico. J Immigr Minor Health in press 2014.

FESTA A, D'AGOSTINO R JR, MYKKÄNEN L, TRACY RP, ZACCARO DJ, HALES CN, HAFFNER SM: Relative contribution of insulin and its precursors to fibrinogen and PAI-1 in a large population with different states of glucose tolerance. The Insulin Resistance Atherosclerosis Study (IRAS). Arterioscler Thromb Vasc Biol 19: $562-568,1999$. 
FINUCANE OM, REYNOLDS CM, MCGILLICUDDY FC, ROCHE HM: Insights into the role of macrophage migration inhibitory factor in obesity and insulin resistance. Proc Nutr Soc 71: 622-633, 2012.

GASEVIC D, CARLSSON AC, LESSER IA, MANCINI GJ, LEAR SA: The association between "hypertriglyceridemic waist" and sub-clinical atherosclerosis in a multiethnic population: a cross-sectional study. Lipids Health Dis 13: 38, 2014.

GUPTA M, SINGH N, TSIQOULIS M, KAIIJ M, HIRJIKALA S, QUAN A, TEOH H, VERMA S: Perceptions of Canadian primary care physicians towards cardiovascular risk assessment and lipid management. Can $J$ Cardiology 28: 14-19, 2012.

HAN KJ, LEE SY, KIM NH, CHAE HB, LEE TH, JANG CM, YOO KM, PARK HJ, LEE MK, JEON WS, PARK SE, PARK CY, LEE WY, OH KW, PARK SW, RHEE EJ: Increased risk of diabetes development in subjects with the hypertriglyceridemic waist phenotype: a 4-year longitudinal study. Endocrinol Metab (Seoul) 29: 514-521, 2014.

HE S, ZHENG Y, SHU Y, HE J, WANG Y, CHEN X: Hypertriglyceridemic waist might be an alternative to metabolic syndrome for predicting future diabetes mellitus. PLoS One 8: e73292, 2013.

HEILBRONN LK, CAMPBELL LV: Adipose tissue macrophages, low grade inflammation and insulin resistance in human obesity. Curr Pharm Des 14: 1225-1230, 2008.

KLEIN S, ALLISON DB, HEYMSFIELD SB, KELLEY DE, LEIBEL RL, NONAS C, KAHN R; ASSOCIATION FOR WEIGHT MANAGEMENT AND OBESITY PREVENTION; NAASO, THE OBESITY SOCIETY; AMERICAN SOCIETY FOR NUTRITION; AMERICAN DIABETES ASSOCIATION: Waist circumference and cardiometabolic risk: a consensus statement from Shaping America's Health: Association for Weight Management and Obesity Prevention; NAASO, The Obesity Society; the American Society for Nutrition; and the American Diabetes Association. Am J Clin Nutr 85: 1197-1202, 2007.

LAMARCHE B, TCHERNOF A, MAURIĖGE P, CANTIN B, DAGENAIS GR, LUPIEN PJ, DESPRÉS JP: Fasting insulin and apolipoprotein B levels and low-density lipoprotein particle size as risk factors for ischemic heart disease. JAMA 279: 1955-1961, 1998.

LEMIEUX I, PASCOT A, COUILLARD C, LAMARCHE B, TCHERNOF A, ALMÉRAS N, BERGERON J, GAUDET D, TREMBLAY G, PRUD'HOMME D, NADEAU A, DESPRÉS JP: Hypertriglyceridemic waist: a marker of the atherogenic metabolic triad (hyperinsulinemia; hyperapolipoprotein B; small, dense LDL) in men? Circulation 102: 179-184, 2000.

LEMIEUX I, POIRIER P, BERGERON J, ALMÉRAS N, LAMARCHE B, CANTIN B, DAGENAIS GR, DESPRÉS JP: Hypertriglyceridemic waist: a useful screening phenotype in preventive cardiology? Can J Cardiol $\mathbf{2 3}$ (Suppl B): 23B-31B, 2007.

MATTHEWS DR, HOSKER JP, RUDENSKI AS, NAYLOR BA, TREACHER DF, TURNER RC: Homeostasis model assessment: insulin resistance and beta-cell function from fasting plasma glucose and insulin concentrations in man. Diabetologia 28: 412-419, 1985.

NATIONAL CHOLESTEROL EDUCATION PROGRAM (NCEP) EXPERT PANEL ON DETECTION, EVALUATION, AND TREATMENT OF HIGH BLOOD CHOLESTEROL IN ADULTS (ADULT TREATMENT PANEL III): Third Report of the National Cholesterol Education Program (NCEP) Expert Panel on Detection, Evaluation, and Treatment of High Blood Cholesterol in Adults (Adult Treatment Panel III) final report. Circulation 106: 3143-3421, 2002.

O'LEARY DH, POLAK JF, KRONMAL RA, MANOLIO TA, BURKE GL, WOLFSON SK JR: Carotid-artery intima and media thickness as a risk factor for myocardial infarction and stroke in older adults. Cardiovascular Health Study Collaborative Research Group. N Engl J Med 340: 14-22, 1999.

PALOMO IG, GUTIÉRREZ CL, ALARCÓN ML, JARAMILLO JC, SEGOVIA FM, LEIVA EM, MUJICA VE, ICAZA GN, DÍAZ NS, MOORE-CARRASCO R: Increased concentration of plasminogen activator inhibitor-1 and fibrinogen in individuals with metabolit syndrome. Mol Med Rep 2: 253-257, 2009.

PINHEIRO VOLP AC, SANTOS SILVA FC, BRESSAN J: Hepatic inflammatory biomarkers and its link with obesity and chronic diseases. Nutr Hosp 31: 1947-1956, 2015. 
REINER Ž, CATAPANO AL, DE BACKER G, GRAHAM I, TASKINEN MR, WIKLUND O, AGEWALL S, ALEGRIA E, CHAPMAN MJ, DURRINGTON P, ERWINE S, HALCOX J, HOBBS R, KJESHUS J, FILARDI PP, RICHARDI G, STOREY RF, WOOD D: ESC/EAS Guideliens for the management of dyslipidemias. The Task Force for the management of dyslipidemias of the European Society of Cardiology (ESC) and the European Atherosclerosis Society (EAS). Eur Heart J 32: 1769-1818, 2011.

SAKKINEN PA, WAHL P, CUSHMAN M, LEWIS MR, TRACY RP: Clustering of procoagulation, inflammation, and fibrinolysis variables with metabolic factors in insulin resistance syndrome. Am J Epidemiol 152: 897-907, 2000.

ST-PIERRE J, LEMIEUX I, PERRON P, BRISSON D, SANTURÉ M, VOHL MC, DESPRÉS JP, GAUDET D: Relation of the "hypertriglyceridemic waist" phenotype to earlier manifestations of coronary artery disease in patients with glucose intolerance and type 2 diabetes mellitus. Am J Cardiol 99: 369-373, 2007.

TANKÓ LB, BAGGER YZ, QIN G, ALEXANDERSEN P, LARSEN PJ, CHRISTIANSEN C: Enlarged waist combined with elevated triglycerides is a strong predictor of accelerated atherogenesis and related cardiovascular mortality in postmenopausal women. Circulation 111: 1883-1890, 2005.

ZHANG M, GAO Y, CHANG H, WANG X, LIU D, ZHU Z, HUANG G: Hypertriglyceridemic-waist phenotype predicts diabetes: a cohort study in Chinese urban adults. BMC Public Health 12: 1081, 2012. 